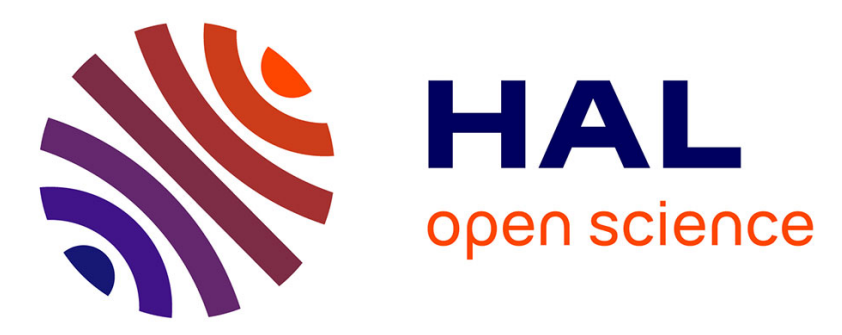

\title{
Personality and memory performance over twenty years: Findings from three prospective studies
}

Yannick Stephan, Angelina R. Sutin, Martina Luchetti, Antonio Terracciano

\section{To cite this version:}

Yannick Stephan, Angelina R. Sutin, Martina Luchetti, Antonio Terracciano. Personality and memory performance over twenty years: Findings from three prospective studies. Journal of Psychosomatic Research, 2020, 128, pp.109885 -. 10.1016/j.jpsychores.2019.109885 . hal-03488468

\section{HAL Id: hal-03488468 \\ https://hal.science/hal-03488468}

Submitted on 21 Dec 2021

HAL is a multi-disciplinary open access archive for the deposit and dissemination of scientific research documents, whether they are published or not. The documents may come from teaching and research institutions in France or abroad, or from public or private research centers.
L'archive ouverte pluridisciplinaire HAL, est destinée au dépôt et à la diffusion de documents scientifiques de niveau recherche, publiés ou non, émanant des établissements d'enseignement et de recherche français ou étrangers, des laboratoires publics ou privés.

\section{다)(1) $(5$}

Distributed under a Creative Commons Attribution - NonCommerciall 4.0 International 


\section{RUNNING HEAD: PERSONALITY AND MEMORY}

Personality and Memory Performance over Twenty Years:

Findings from Three Prospective Studies

Yannick Stephan ${ }^{1 *}, \mathrm{PhD}$, Angelina R. Sutin ${ }^{2}, \mathrm{PhD}$, Martina Luchetti ${ }^{2}, \mathrm{PhD}$, Antonio Terracciano ${ }^{3}, \mathrm{PhD}$

${ }^{1}$ Euromov, University of Montpellier, FRANCE

2 Department of Behavioral Sciences and Social Medicine, College of Medicine, Florida State University, USA

${ }^{3}$ Department of Geriatrics, College of Medicine, Florida State University, USA

Word count: 2736 words

\section{Tables: 2}

Correspondence concerning this article should be addressed to Yannick Stephan, Euromov, University of Montpellier, UFRSTAPS, 700, Avenue du Pic St Loup, 34090 Montpellier, France. E-mail: yannick.stephan@umontpellier.fr

Angelina R. Sutin: angelina.sutin@ med.fsu.edu

Martina Luchetti : martina.luchetti@med.fsu.ed

Antonio Terracciano : antonio.terracciano@med.fsu.edu 


\section{Abstract}

Objective. The present study examined whether personality traits are related to episodic memory over the long-term. Method Participants were adults from the Wisconsin Longitudinal Study Graduate (WLSG, N= 3,726) and Sibling samples (WLSS, $N=1,720$ ), and the Midlife in the United States Study (MIDUS, N=2,411). Five factor model personality traits and demographic factors were measured at baseline. Memory performance on immediate and delayed free recall tasks was assessed at follow-up, almost 20 years later. Results. In regression models that accounted for demographic factors, consistent evidence was found across three samples that middle-aged adults who scored higher on neuroticism performed significantly worse on a memory test 20 years later. In the WLSG and WLSS and a meta-analysis, higher openness was also associated with better memory at follow-up. High neuroticism and low openness were also associated with a 20 to $40 \%$ increased risk of performing below one and a half standard deviation from the sample mean on the memory task. Conclusions. The present study extends previous research with evidence that the association between personality traits and memory function persist over two decades.

Key-words: Personality, memory, prospective studies 


\section{Introduction}

The identification and understanding of factors that shape memory function with aging is of crucial importance for the health and well-being of older adults. Memory impairment predicts functional decline [1], higher risk of dementia [2], and earlier mortality [3]. Among a range of potential influential factors, a growing body of research indicates that personality contributes to memory in old age. Specifically, higher neuroticism (the propensity to experience distress and negative affect) is related to lower episodic memory performance, whereas higher openness (the preference for variety and intellectual curiosity) and conscientiousness (the tendency to be self-disciplined and organized) are associated with better memory function [4-7]. Less consistent associations have been observed between episodic memory performance and both extraversion (the propensity to be active and to experience positive emotions) and agreeableness (the tendency to be altruistic and trusting) [7].

Consistent with cross-sectional findings, longitudinal research suggests that higher openness and conscientiousness mitigate memory decline, whereas higher neuroticism is related to a steeper decline $[7,8,9]$. These studies have examined the association between personality and memory over follow-up intervals ranging from 4 to 14 years, and the extent to which this association persists over longer period is unknown. The longest study to date had a follow-up of 14 years and examined only neuroticism [5]. There are reasons to expect that personality may predict memory over longer periods. Personality traits, for example, have been found to predict risk of incident Alzheimer's disease over 20 years or more $[10,11]$. Given that memory deficits are a core feature of Alzheimer's disease, the association between personality and memory may persist over several decades.

Using data from three large national samples of middle aged and older adults, the present study examines the prospective association between personality and episodic memory 
assessed twenty years later. Episodic memory was assessed using immediate and delayed free recall tasks. In line with existing cross-sectional and longitudinal findings, it was hypothesized that higher neuroticism is related to lower memory performance at follow-up. In contrast, higher openness and conscientiousness were expected to be associated with higher memory function. In addition, given that these traits have been related to higher likelihood of $\mathrm{AD}[10,11]$, higher neuroticism, lower openness and conscientiousness were expected to relate to higher likelihood of memory impairment, defined as a score equal to or lower than one and half standard deviations below the sample mean [12].

\section{Method}

\subsection{Participants}

Data from the Wisconsin Longitudinal Study (WLS) and from the Midlife in the United States Survey (MIDUS) were used in the present study. Participants with complete data on demographic information, including age, sex, education and race (MIDUS only), and personality at baseline and memory at follow-up were included in each sample. All participants provided written informed consent. Descriptive statistics for the three samples are presented in Table 1.

The Wisconsin Longitudinal Study is a long-term study of a random sample of 10,317 men and women who graduated from Wisconsin high schools in 1957 (WLSG). The WLS sample is broadly representative of white, non-Hispanic American men and women who have completed at least a high school education. Personality and demographic data were obtained in 1992-1993 from a total of 6,673 participants. Of this sample, 3,726 participants aged from 51 to 56 years old also provided memory data in 2011 and were included as the final analyzed sample $(54 \%$ women, mean age $=53.18, \mathrm{SD}=0.60)$. Selected siblings $(\mathrm{WLSS})$ of some of the graduates are also included in the WLS. A total of 3,426 individuals provided data on personality and demographic factors in 1993-1994. The final sample was composed of 1,720 
participants aged from 29 to 75 years old at baseline who also provided complete memory data in $2011(54 \%$ women, mean age $=52.44, \mathrm{SD}=6.90)$. In the WLSG, participants without memory data at follow-up were older, $t(6671)=4.73, p<.001$ and had less education, $t(6671)=6.35, p<.001$. In addition, they had higher neuroticism, $t(6671)=4.93, p<.001$ and lower extraversion, $t(6671)=2.35, p<.05$, openness, $t(6671)=3.36, p<.001$, and agreeableness, $t(6671)=2.09, p<.05$. No differences were found for conscientiousness, $t(6671)=0.65, p=.51$, and sex, $\chi^{2}(1,6673)=0.34, p=.56$. In the WLSS, participants without memory data at follow-up were older, $t(3424)=8.81, p<.001$, had less education, $t(3424)=$ $5.90, p<.001$, and had lower openness, $t(3424)=2.56, p<.05$. No differences were found for extraversion, $t(3424)=1.43, p=.15$, agreeableness, $t(3424)=1.85, p=.06$, neuroticism, $t(3424)=0.91, p=.36$, conscientiousness, $t(3424)=0.32, p=.75$, and sex, $\chi^{2}(1,3426)=1.83$, $p=.17$.

The MIDUS is a sample of non-institutionalized, English-speaking adults. Data were drawn from the first (1994-1995, MIDUS I) and third (2013-2014, MIDUS III) waves. Complete baseline demographic and personality data were obtained from 6,075 participants. The final analyzed sample was composed of 2,411 individuals aged from 29 to 75 years old who also provided memory data at follow-up $(55 \%$ women, mean age $=45.78, \mathrm{SD}=11.11)$. In the MIDUS, participants without memory data at follow-up were older, $t(6073)=5.23, p<$ .001 , had less education, $t(6073)=15.11, p<.001$, were less likely to be white, $\chi^{2}(1,6075)=$ 63.07, $p<.001$, more likely to be male, $\chi^{2}(1,6075)=8.01, p<.01$, and had higher neuroticism, $t(6073)=4.14, p<.001$, lower conscientiousness, $t(6073)=5.27, p<.001$, and lower openness $t(6073)=2.57, p<.05$. No differences were found for agreeableness, $t(6073)=0.86, p=.39$ or extraversion, $t(6073)=1.20, p=.23$.

\subsection{Personality}


Participants in the WLSG and WLSS samples completed a 29-item version of the Big Five Inventory (BFI) [13]. Participants were asked whether they agreed or disagreed with descriptive statements assessing neuroticism (e.g. "To what extent do you agree that you see yourself as someone ... who worries a lot?”), extraversion (e.g., “...is talkative”), openness (e.g., “...values artistic, aesthetic experiences”), agreeableness (e.g., “...who is generally trusting”) and conscientiousness (e.g., “...who does things efficiently”). Answers were given using a 6-point rating scale, ranging from 1 (disagree strongly) to 6 (agree strongly). In the MIDUS, the Midlife Development Inventory (MIDI) was used [14]. The MIDI includes 26 adjectives that assess neuroticism (e.g., "nervous"), extraversion (e.g., "friendly"), openness (e.g., "creative"), agreeableness (e.g., "caring"), and conscientiousness (e.g., "organized”). Participants were asked how much each adjective described them on a scale ranging from 1 (not at all) to 4 (a lot). Cronbach alphas for the WLG, the WLSS, and the MIDUS were, respectively, .77, .75, and .75 for neuroticism, .77, .77, and .77 for extraversion, $.62, .59$ and .74 for openness, .68, .68, and .81 for agreeableness, and .63, .66, and .57 for conscientiousness.

\subsection{Episodic Memory.}

In the MIDUS, participants were asked to listen to a list of 15 words and then to recall as many words as possible both immediately and after a delay of approximately 12 minutes. In both the WLSG and WLSS, participants were asked to repeat as many words as they could from a list of 10 words, both immediately and after a delay of approximately 5 minutes. In the three samples, the number of correct words for immediate and delayed recall were summed to give a memory performance score.

\subsection{Covariates.}

The analysis controlled for age, sex, and education in the three samples because these demographic factors are likely to influence memory performances [15]. Education was 
reported in years in both the WLSG and the WLSS, and on a scale from 1 (no grade school) to 12 (doctoral level degree) in the MIDUS. Because of its effect on memory [15], race was an additional covariate in the MIDUS.

\subsection{Data analysis}

Linear regression analyses were conducted to examine the relation between personality and memory. In each sample, memory performance at follow-up was predicted by each personality trait, controlling for age, sex, education, and race (MIDUS only). Results from the three samples were combined in a random effects meta-analysis with Comprehensive Meta-Analysis software. Finally, the association between personality and memory impairment, defined as a memory performance equal to or lower than 1.5 SD below the mean [12], was tested with logistic regressions, with the traits as predictors of likelihood of memory impairment, controlling for demographic factors. In both the WLSG and WLSS, IQ in adolescence was included as covariate in additional analysis.

\section{Results}

Descriptive statistics indicated that the three samples were relatively similar for sex and race, and that the MIDUS was slightly younger than the WLS samples (Table 1). In all three samples, there was consistent evidence that higher neuroticism at baseline was associated significantly with worse memory performance almost 20 years later (see Table 2). Higher neuroticism accounted for $0.1 \%, 0.2 \%$ and $0.5 \%$ of the memory variance in the WLSG, WLSS, and MIDUS, respectively, controlling for demographic factors. The difference in memory performance between the high (top quartile) and low (bottom quartile) neuroticism was $d=.11$ and $d=.12$ for the WLSG and the WLSS, respectively, and $d=.16$ for the MIDUS. In addition, higher openness was associated with better memory in the WLS samples and explained, respectively, $0.2 \%$ and $0.4 \%$ of variance in memory in the WLSG and 
WLSS. The difference in memory performance between high (top quartile) and low (bottom quartile) openness was $d=.12$ for the WLSG and $d=.13$ for the WLSS. Conscientiousness, extraversion, and agreeableness were not significant predictors of memory performance. The overall pattern of associations was similar across samples, with little evidence of heterogeneity (Table 2). In the WLSG and the WLSS, the pattern of associations changed when IQ was included in the analysis. Indeed, the association between neuroticism and memory was no longer significant in both the WLSG $(\beta=-.02, p=.23)$ and the WLSS $(\beta=-.05$, $p=.06)$. Furthermore, openness was no longer significantly related to memory in both samples $\left(\beta_{\mathrm{WLSG}}=.02, p=.12 ; \beta_{\mathrm{WLSS}}=.04 ; p=.16\right)$.

Finally, we examined the association between personality and memory impairment. There were 228 participants $(6 \%)$ in the WLSG, 113 participants in the WLSS (7\%), and 154 participants $(6 \%)$ in the MIDUS who had memory performance $1.5 \mathrm{SD} \leq$ the mean. Logistic regression analysis revealed that neuroticism was related to the risk of memory scores $\leq 1.5$ SD in both the WLSS and the WLSG: one standard deviation higher score on neuroticism was related to an approximately $20-40 \%$ higher risk of memory $\leq 1.5$ SD in the WLSG (odds ratio $=1.19,95 \%$ CI: 1.04-1.36, $\mathrm{p}<.05$ ) and WLSS (odds ratio= 1.40, 95\% CI: 1.14-1.71, $\mathrm{p}<.01)$. In addition, one SD higher score on openness was associated with a $25 \%$ reduced risk of memory $\leq 1.5 \mathrm{SD}$ in the WLSS (odds ratio=0.74, 95\% CI: 0.59-0.93, p<.05). Conscientiousness, extraversion, and agreeableness were not associated with risk of memory scores $\leq 1.5$ SD. No significant associations were found in the MIDUS. When IQ was included as a covariate, neuroticism remained significantly related to memory impairment in both the WLSG (odds ratio $=1.15,95 \%$ CI: 1.01-1.32, $\mathrm{p}<.05$ ) and the WLSS (odds ratio= 1.38, 95\% CI: 1.12-1.71, $\mathrm{p}<.01$ ), and the relationship between openness and memory impairment persisted in the WLSS (odds ratio= 0.78, 95\% CI: 0.62-0.98, p<.05).

\section{Discussion}


Using three large longitudinal survey of middle-aged and older individuals $(\mathrm{N}>$ $7,000)$, the present study examined the long-term associations between personality traits and episodic memory function. Consistently across the three samples, higher neuroticism was related to lower memory performance assessed almost 20 years later. In addition, higher openness was associated with better episodic memory in two of three samples and in the meta-analysis. Contrary to past research, however, conscientiousness was not a significant predictor of memory performance. The present study extends existing research by providing the longest prospective evidence of an association between personality traits and episodic memory.

The consistent association between neuroticism and memory performance assessed two decades later supports the hypothesis and extends existing cross-sectional and shorterterm longitudinal research [4-9]. In addition, higher neuroticism was associated with higher likelihood of memory impairment, defined as performance 1.5SD below the mean. There may be behavioral, affective, and biological implications of neuroticism that may be deleterious for memory function not only in the short-term but also over at least two decades. Neuroticism, for example, is related to higher stress sensitivity [16] and physiological dysregulation [17], which may alter memory function decades later. In addition, higher neuroticism is related to higher depressive symptoms [18], sleeping difficulties [19], and to health-risk behaviors, such as smoking [20], physical inactivity [21] and alcohol use [22], which may have long-term implications for memory.

As expected, higher openness to experience was related to better long-term memory performance. Higher openness was also associated with a lower likelihood of memory impairment in one sample. This result adds to past research having found a protective role of openness for memory over shorter time spans [7]. Openness to experience is associated with cognitively stimulating activities, such as cultural [23] and physical activities [21]. This active 
and cognitively stimulating lifestyle may have a cumulative beneficial effect for memory over the long term. Furthermore, higher openness is related to lower inflammation [24], which may also contribute to better memory.

In contrast to our hypothesis, conscientiousness was not related to memory across the three samples. This finding suggests that the memory benefits of this trait observed in cross-sectional and short-term longitudinal research [4,7] may dissipate over time. The lack of significant association between conscientiousness and memory over 20 years stands in contrast with studies that find an association between higher conscientiousness and lower AD risk over two decades $[10,11]$. Taken together, past studies combined with the present results suggest that conscientiousness may be more strongly related to complex cognitive dimensions implicating a myriad of cognitive processes over the long term, than to single measures assessing one type of ability.

Unexpectedly, the meta-analysis revealed also that higher extraversion was related to better memory at follow-up. This finding contrasts with existing research that found longitudinal associations between higher extraversion and lower memory performance [7]. However, it is close to a recent report of a positive cross-sectional link between extraversion and delayed recall [4]. Extraversion is related to memory-enhancing pathways, such as frequent social interactions [25], reduced reactivity to stressors [16], better sleep quality [19], and a physically active lifestyle [21], which may accumulate to benefit long-term memory performance. Consistent with existing research, agreeableness was unrelated to memory performance.

The present study has several strengths including the identification of the prospective association between personality and memory across three large samples of middle-aged and older adults, over a follow-up of almost 20 years, and a meta-analysis. However, there are also limitations to consider. The observational design of the present study 
limits the ability to conclude causal relations between personality and memory. With such a design, it is only possible to test for a correlation between personality and memory. Therefore, although personality was tested as a predictor of memory, reciprocal associations may also exist. In this study, baseline assessments of memory function were lacking, and it was not possible to examine the association between personality and change in memory over time. Furthermore, the generalizability of our findings is limited by the positive selection of the three samples. The results were also specific to episodic memory. Future research must test whether the associations observed extend to other types of memory. The size of the association between personality and memory was relatively small. However, this size must be considered with regard to the 20-year follow-up period of the present study. In addition, memory is multi-determined and reflects a wide range of factors, each of which may only have a relatively small effect size. In addition, personality is a distal predictor that acts through behavioral, affective and biological factors that are proximal to memory function. Further research is needed to examine the mediating role of these factors in the link between personality and episodic memory. The present study only controlled for age, sex, education and race, but the results were potentially influenced by other confounding factors such as baseline memory or dementia status, which were not available in the three samples. Future research should account for possible confounding and mediating factors. Finally, future research should include a facet-level analysis of the association between personality and longterm memory.

Despite these limitations, the present study provides new evidence of the long-term association between personality and memory across adulthood. From a practical perspective, this finding suggests that personality assessments may prove useful to identify individuals at risk of long-term memory difficulties and who may benefit from interventional efforts. 
Acknowledgment.

The Wisconsin Longitudinal Study (WLS) has been supported principally by the National Institute on Aging (AG-9775, AG-21079, AG-033285, and AG-041868), with additional support from the Vilas Estate Trust, the National Science Foundation, the Spencer Foundation, and the Graduate School of the University of Wisconsin-Madison. The Midlife in the United States study 1 (MIDUS 1) was supported by the John D. and Catherine T. MacArthur Foundation Research Network on Successful Midlife Development. The MIDUS 2 and MIDUS 3 research was supported by grants from the National Institute on Aging (P01AG020166, R37AG027343). Additional grants were obtained from grants from the General Clinical Research Centers Program (M01-RR023942, M01-RR00865) and the National Center for Advancing Translational Sciences (UL1TR000427). This research was also supported from the National Institute on Aging of the National Institutes of Health, Award Numbers R21AG057917 and R01AG053297. 


\section{References}

1. Hooghiemstra AM, Ramakers IH, Sistermans N, et al.: Gait speed and grip strength reflect cognitive impairment and are modestly related to incident cognitive decline in memory clinic patients with subjective cognitive decline and mild cognitive impairment: Findings from the 4C study. J Gerontol A Biol Sci Med Sci. 2017; 72: 846-854.

2. Aggarwal NT, Wilson RS, Beck TL, et al.: Mild cognitive impairment in different functional domains and incident Alzheimer's disease. J Neurol Neurosurg Psychiatry 2005; 76: $1479-1484$.

3. Batty GD, Deary IJ, Zaninotto P.: Association of cognitive function with cause-specific mortality in middle and older age: Follow-up of participants in the English Longitudinal Study of Ageing. Am J Epidemiol. 2016; 183: 183-190.

4. Chapman BP, Benedict RH, Lin F., et al.: Personality and performance in specific neurocognitive domains among older persons. Am J Geriatr Psychiatry 2017; 25: 900-908.

5. Klaming R, Veltman DJ, Comijs HC.: The impact of personality on memory function in older adults—results from the Longitudinal Aging Study Amsterdam. Int J Geriatr Psychiatry 2017; 32:798-804.

6. Graham EK, Lachman ME.: Personality stability is associated with better cognitive performance in adulthood: Are the stable more able?. J Gerontol B Psychol Sci Soc Sci. 2012; 67: 545-554.

7. Luchetti M, Terracciano A, Stephan Y, et al.: Personality and cognitive decline in older adults: Data from a longitudinal sample and meta-analysis. J Gerontol B Psychol Sci Soc Sci. 2016 ; 71: 591-601.

8. Caselli RJ, Dueck AC, Locke DE, et al.: Impact of personality on cognitive aging: A prospective cohort study. J Int Neuropsychol Soc. 2016 ; 22: 765-776. 
9. Hock RS, Lee HB, Bienvenu OJ, et al.: Personality and cognitive decline in the Baltimore Epidemiologic Catchment Area follow-up study. Am J Geriatr Psychiatry 2014 ; 22: 917925.

10. Terracciano A, Sutin AR, An Y et al.: Personality and risk of Alzheimer's disease: New data and meta-analysis. Alzheimers Dement. 2014 ; 10: 179-186.

11. Johansson L, Guo X, Duberstein PR, et al. : Midlife personality and risk of Alzheimer disease and distress : A 38-year follow-up. Neurology 2014 ; 83: 1538-1544.

12. Kasper JD, Freedman VA, Spillman BC.: Classification of persons by dementia status in the National Health and Aging Trends Study. Baltimore, MD: Johns Hopkins University School of Public Health; 2014.

13. John OP, Donahue EM, Kentle RL.: The Big Five Inventory-Versions 4a and 54. Berkeley, CA: Institute of Personality and Social Research, University of California 1991.

14. Zimprich D, Allemand M, Lachman ME.: Factorial structure and age-related psychometrics of the MIDUS personality adjective items across the lifespan. Psychol Assess 2012 ; 24: 173-186.

15. Rickenbach EH, Agrigoroaei S, Lachman ME. Awareness of memory ability and change: (In)Accuracy of memory self-assessments in relation to performance. J Popul Ageing. 2015;8(1-2):71-99. doi:10.1007/s12062-014-9108-5

16. Leger KA, Charles ST, Turiano NA, et al.: Personality and stressor-related affect. J Pers Soc Psychol. 2016; 111:917-928. 
17. Sutin AR, Stephan Y, Terracciano A.: Personality and metabolic dysfunction in young adulthood: A cross-sectional study. J Health Psychol. 2019; 24:495-501.

18. Hakulinen C., Elovainio M, Pulkki-Råback L, et al.: Personality and depressive symptoms: Individual participant meta-analysis of 10 cohort studies. Depress Anxiety 2015; 32: 461-470.

19. Stephan Y, Sutin AR, Bayard S, et al.: Personality and sleep quality: Evidence from four prospective studies. Health Psychol. 2018; 37:271-281.

20. Hakulinen C, Hintsanen M, Munafo MR, et al.: Personality and smoking: Individualparticipant meta-analysis of nine cohort studies. Addiction. 2015; 110:1844-1852.

21. Sutin AR, Stephan Y, Luchetti M., et al.: The five-factor model of personality and physical inactivity: A meta-analysis of 16 samples. J Res Pers. 2016; 63:22-28.

22. Hakulinen C, Elovainio M, Batty GD, et al.: Personality and alcohol consumption: Pooled analysis of 72,949 adults from eight cohort studies. Drug Alcohol Depend. 2015; 151:110114.

23. Schwaba T, Luhmann M, Denissen JJA, et al.: Openness to experience and cultureopenness transactions across the lifespan. J Pers Soc Psychol. 2018; 115:118-136.

24. Luchetti M, Barkley JM, Stephan Y, et al.: Five-factor model personality traits and inflammatory markers: New data and a meta-analysis. Psychoneuroendocrinology. 2014; 50:181-93.

25. Stephan Y, Boiché J, Canada B,et al.: Association of personality with physical, social, and mental activities across the lifespan: Findings from US and French samples. Br J Psychol. 2014;105:564-580. 
Table 1.

Characteristics of the Samples at Baseline and Memory Performance at Follow-Up.

\begin{tabular}{lcccccc}
\hline & \multicolumn{2}{c}{ WLSG } & \multicolumn{2}{c}{ WLSS } & \multicolumn{2}{c}{ MIDUS } \\
\hline Variables & $M / \%$ & $S D$ & $M / \%$ & $S D$ & $M / \%$ & $S D$ \\
\hline Age (Years) & 53.18 & 0.60 & 52.44 & 6.90 & 45.78 & 11.11 \\
Sex (\% women) & $54 \%$ & - & $54 \%$ & - & $55 \%$ & - \\
Race (\% White) & $100 \%$ & - & $100 \%$ & - & $95 \%$ & - \\
Education & 13.87 & 2.40 & 14.03 & 2.51 & 7.45 & 2.45 \\
Neuroticism & 3.15 & 0.97 & 3.21 & 0.94 & 2.19 & 0.66 \\
Extraversion & 3.86 & 0.89 & 3.78 & 0.90 & 3.21 & 0.55 \\
Openness & 3.67 & 0.80 & 3.64 & 0.75 & 3.04 & 0.50 \\
Agreeableness & 4.76 & 0.73 & 4.71 & 0.72 & 3.48 & 0.49 \\
Conscientiousness & 4.87 & 0.67 & 4.77 & 0.71 & 3.46 & 0.43 \\
Memory Performance & 9.00 & 2.90 & 9.07 & 3.07 & 11.21 & 4.73 \\
\hline
\end{tabular}

Note. WLSG: $\mathrm{N}=3,726$; WLSG: $\mathrm{N}=1,720$; MIDUS: $\mathrm{N}=2,411$

See method section for differences in the assessment of memory, personality, and education in each sample. 
Table 2

Regression Analysis Predicting Follow-up Memory Performance from Baseline Personality

\begin{tabular}{lccccc}
\hline & Neuroticism & Extraversion & Openness & Agreeableness & Conscientiousness \\
\hline WLSG $^{\mathrm{a}}$ & $-.04 *$ & .01 & $.04 *$ & -.01 & -.01 \\
& & & & & \\
& & & & \\
$\mathrm{WLSS}^{\mathrm{a}}$ & $-.05^{*}$ & .04 & $.07 * *$ & .02 & .02 \\
MIDUS $^{\mathrm{b}}$ & $-.07 * * *$ & .03 & .03 & -.03 & -.03 \\
Meta-analysis & -.05 & .02 & .05 & -.01 & $(-.035 ; .022)$ \\
Random Effect & $(-.082 ;-.028)^{* * *}$ & $(.001 ; .046)^{*}$ & $(.024 ; .068)^{* * *}$ & $(-.035 ; .014)$ & $37 \%$ \\
Heterogeneity I $^{2}$ & $31 \%$ & $0 \%$ & $0 \%$ & $17 \%$ & \\
\hline
\end{tabular}

Note. WLSG: $\mathrm{N}=3,726$; WLSS: $\mathrm{N}=1,720$, MIDUS: $\mathrm{N}=2,411$

Coefficients are standardized coefficients

${ }^{a}$ Adjusted for age, sex, and education.

${ }^{\mathrm{b}}$ Adjusted for age, sex, education, and race.

$* p<.05, * * p<.01, * * * p<.001$ 